\title{
Multimodality endoscopic imaging technology for visualization of layered architecture and vasculature
}

\section{Yan Li, Zhongping Chen}

Yan Li, Zhongping Chen, "Multimodality endoscopic imaging technology for visualization of layered architecture and vasculature," Proc. SPIE 11052, Third International Conference on Photonics and Optical Engineering, 110520B (24 January 2019); doi: 10.1117/12.2520537

SPIE Event: The International Conference on Photonics and Optical Engineering, 2018, Xi'an, China 


\title{
Multimodality Endoscopic Imaging Technology for Visualization of Layered Architecture and vasculature
}

\author{
Yan Li ${ }^{1}$ and Zhongping Chen ${ }^{1,2, *}$ \\ ${ }^{1}$ Beckman Laser Institute, University of California, Irvine, 1002 Health Sciences Road, Irvine, CA \\ 92617 USA \\ ${ }^{2}$ Department of Biomedical Engineering, University of California Irvine, Irvine, CA 92697-2700 \\ USA \\ ${ }^{3}$ California Institute of Technology, 1200 E. California Blvd., Pasadena, CA 91125 USA \\ *z2chen@uci.edu
}

\begin{abstract}
Endoscopic imaging technologies, such as endoscopic optical coherence tomography (OCT), near infrared (NIR) fluorescence, photoacoustic (PA), and ultrasound (US) have been used to investigate vascular and morphological changes as hallmarks of early cancer in the gastrointestinal (GI) tract. Here, we developed two multimodality imaging systems which are integrated PA/US and integrated OCT/NIR fluorescence which can obtain layered architecture and vasculature simultaneously. In vivo imaging of rectum wall from Sprague Dawley (SD) rats with these two imaging systems were demonstrated. Both imaging systems enable the use of one imaging probe for performing two different imaging, thereby improving prognosis by early detection and reducing costs. For integrated PA/US, the architectural morphology and vasculature of the rectum wall were visualized without the usage of contrast agent, but slow imaging speed and usage of match medium are the main limitations for clinical translation. With regard to the integrated OCT/NIR fluorescence, it is able to perform high speed imaging, however the addition of contrast agent and limited imaging depth are the main concern for clinical application.
\end{abstract}

Key words: optical coherence tomography, infrared fluorescence, photoacoustic, ultrasound, multimodality, layered architecture, subsurface Microvasculature, gastrointestinal tract 


\section{Introduction}

Globally, there are $\sim 1.4$ million new cases and 694,000 deaths from colorectal cancer annually, which is the third most common type of cancer consisting of $10 \%$ of all cancer cases[1]. Previously, a clinician routinely applies endoscopy to visualize the rectum wall for diagnosis of the $\mathrm{Gl}$ disease[2]. Due to the lack of depth information and limited sensitivity for the vascular lesion, the disorders in which early epithelial dysplastic changes are usually not clearly visible at endoscopy. In recent decades, more and more novel endoscopic imaging technologies[3-9] have been developed to visualize the sub-layered morphology and subsurface vascular network for providing necessary information in order to assess disease stage or progress and plan treatment therapies. For example, endoscopic ultrasound (EUS) $[4,10]$ imaging allows a clinician to obtain images of the $\mathrm{Gl}$ tract and the surrounding tissue and organs with an imaging depth of $\sim 7 \mathrm{~mm}$ and a resolution of $\sim 150 \mu \mathrm{m}$. Endoscopic photoacoustic (PA) imaging is a non-invasive imaging modality that provides structural, functional and molecular information while maintaining a deep depth [11-12]. Endoscopic ОСТ[13-15] which is capable of providing high-resolution cross-sectional images architectural- and cellular-tissue microstructure has been widely applied to image of the esophagus, stomach, small and large intestine, and biliary and pancreatic ducts. In addition, endoscopic Doppler OCT technology $[14,16,17]$ has been applied in endoscopic imaging to visualize vascular networks without any exogenous fluorescent agent. However, limited by the phase stability of the system and movement from the imaging probe, the reconstructed Doppler OCT images often contain motion artifacts and have relatively low sensitivity for microvasculature. Another endoscopic angiography technology is NIR fluorescence imaging[3, 5, 18-20] with indocyanine green (ICG), which provides high resolution microvasculature imaging and has been used clinically to observe a variety of vascular lesions, such as detecting abnormal submucosal vascularization in tumor lesions. By using different kinds of endoscopic imaging technology, the clinician is able to obtain various features of the biological tissue [6]. Therefore, in the clinic, several endoscopic imaging technologies are often needed to obtain enough features of the biological tissue for accurate diagnosis. The usage of different technologies increases the cost and time of imaging for hospitals and patients.

In our study, two multimodality endoscopic imaging systems were developed to investigate $\mathrm{GI}$ lumen morphology and vasculature. For integrate PA/US imaging system, it is able to perform PA 
and US imaging simultaneously at the imaging speed of 20 frames/s. Regarding the integrated OCT/NIR fluorescence, we apply ICG for fluorescence imaging. A B-scan rate up to $100 \mathrm{~Hz}(1000 \mathrm{~A}$ lines/B-scan) was achieved. The results from both imaging system indicate that the multimodality imaging system allows for better identification of the layered morphology and vascular network of the GI tract, which may provide new opportunities for detecting and monitoring GI diseases.

\section{Method}

\subsection{Integrated PA/US imaging system and probe design}

Figure 1 illustrates the overall setup of the integrated PA/US imaging system (a), schematic (b), and (c) photograph of our imaging probe. For PA imaging, a 532-nm nanosecond laser is used for excitation. The output laser beam is coupled by a lens into the multimode fiber (MMF) of the imaging probe. For ultrasound imaging, a single-element transducer is used to detect the photoacoustic and ultrasound signals. The generated PA and US signals are band-pass filtered, amplified, and digitized with a data acquisition (DAQ) card in a personal computer.

In the probe, the laser beam propagates through the MMF, collimated by a 1-mm Gradient index (GRIN) lens, and reflected by a rod mirror with a diameter of $1 \mathrm{~mm}$ at an angle of $45^{\circ}$ towards the tissue surface. Both transducer and rod mirror are tilted slightly in order to obtain the optimal overlap between optical and acoustic beams.

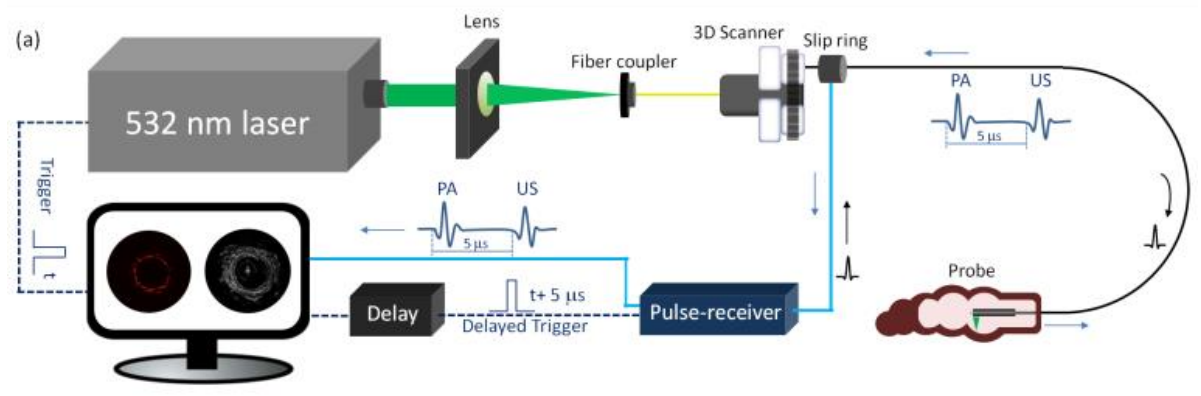

(b)

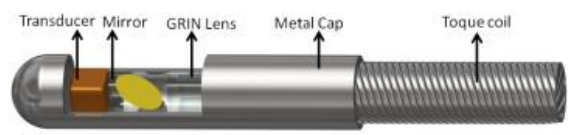

(c)

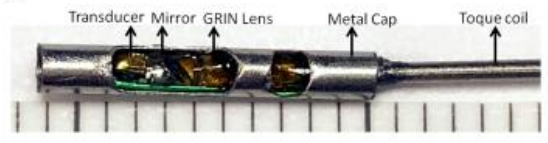

Fig. 1. The setup of integrated imaging system (a), schematic (b), and photography (c) of our imaging probe. 3D scanner consists of fiber optic rotary joint, slip ring, motor, and pull-back translation stage.

\subsection{Integrated OCT/NIR fluorescence imaging system and probe design}

Figure 2 illustrates the overall setup of the integrated OCT and NIR fluorescence imaging system. A wavelength division multiplexer is utilized to combine the OCT and fluorescence laser beams together. For OCT, a $1310 \mathrm{~nm}$ swept source laser is used. For fluorescence imaging, a 785-nm 
semi-conductive CW laser is applied as the excitation source, which corresponds to the excitation peak of the ICG. A double clad fiber (DCF) coupler is incorporated to transmit excitation light and collect emission light.

In the probe, the combined beams propagate through the single mode core of the DCF, focused by a 1 Grin-lens and reflected by a rod mirror at an angle of $45^{\circ}$, then to the tissue surface. A micromotor was used to rotate the rod mirror at a frame rate of 100 images per second.

(a)
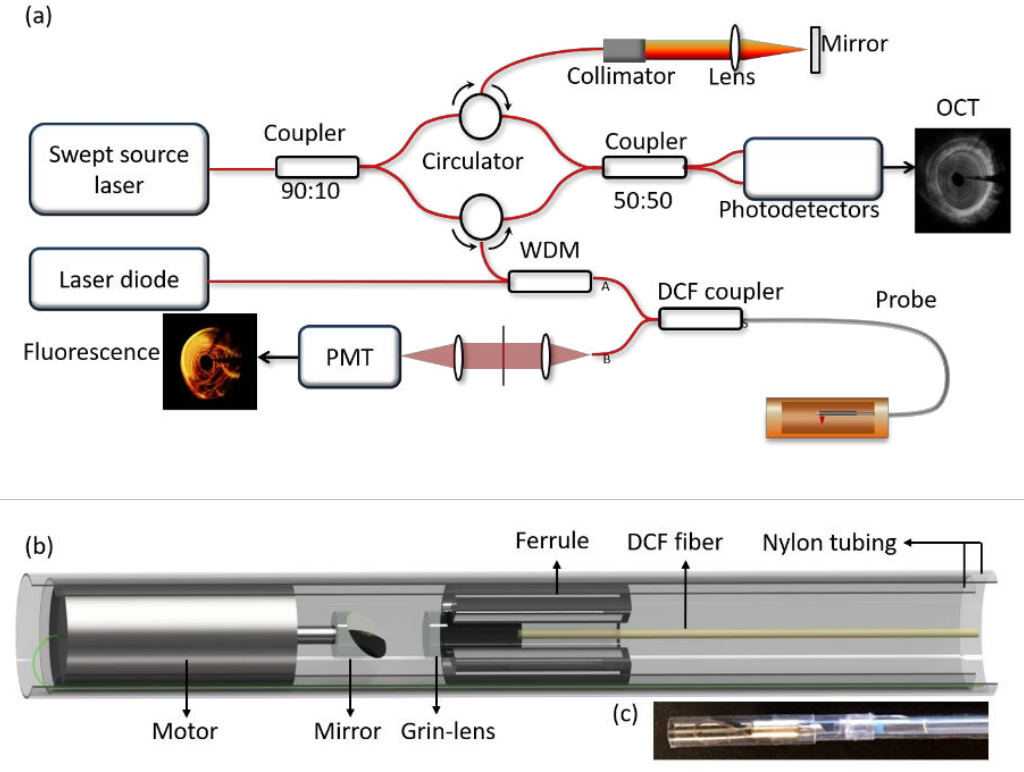

Figure 2 Overall design of the multimodality system. WDM: wavelength division multiplexer. PMT:

photomultiplier tube. DCF coupler: double clad fiber coupler. (b) Overall schematics and (b) photo of the imaging probe.

\section{Results}

We imaged the rectum of a SD rat. The rat was placed in a closed plexiglass chamber into which $5 \%$ isoflurane in oxygen was allowed to flow in order to induce general anesthesia for restraint during the procedure. Following the gas anesthetic induction, the rat was removed from the chamber and an IP injection of ketamine hydrochloride $(87 \mathrm{mg} / \mathrm{kg})$ and xylazine $(10 \mathrm{mg} / \mathrm{kg})$ was administered through a $27 \mathrm{G}$ needle. After the rat was anesthetized, we performed enemas to clean the rectum. For PA/US imaging, we inject sterile distilled water as match medium to perform imaging. For OCT/NIR fluorescence imaging, ICG $(1.5 \mathrm{mg} / \mathrm{Kg})$ was injected via tail vein to target blood vessels of the rectum wall. All experimental protocols were approved by the $\mathrm{UCI}$ IBC under protocol \#2016-3198. 


\subsection{OCT/NIR fluorescence imaging results}

Figures 3 shows the representative combined OCT (inner) and fluorescence (outer) B-scan images of the rectum. From the OCT images, the typical layered architecture can be identified. In addition, several black holes were found, which corresponds to blood vessels. The black shadow at the 8 o'clock direction was caused by the cable of the micromotor. For fluorescence imaging, the region which contains blood vessel will correspond to a high intensity in the fluorescence images.

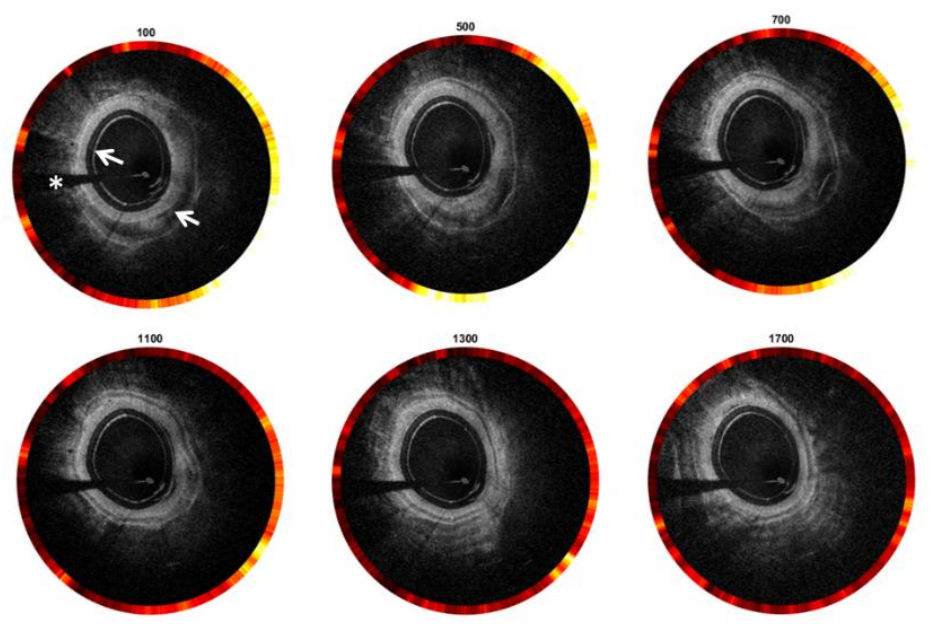

Figure 3 Cross-sectional combined OCT and fluorescence images. White * : micromotor cable; White arrows: plastic sheath and blood clot.

\subsection{PA/US imaging results}

Figure 4 shows the representative US, PA, and combined PA/US images. The typical layered architecture indicated by white dashed box can be found. From the PA images, the signal of blood vessels can be found. Combined PA and US images provide the co-registration images, which will be advantageous over either modality alone to supplement lesion evaluation. 


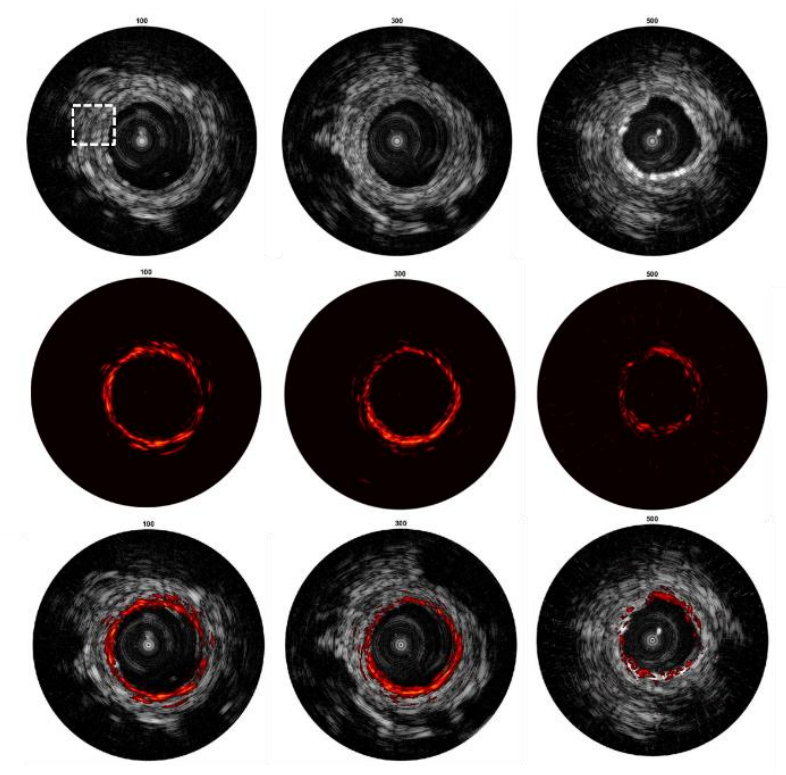

Figure 4 Cross-sectional combined PA and US images.

\section{Discussion}

Multimodality endoscopic OCT/NIR fluorescence imaging and PA/US are non-invasive, nonionizing imaging technology which have the poetical for the diagnosis and arrangement of $\mathrm{Gl}$ disease. For integrated OCT and fluorescence imaging, it provides architectural morphology and molecular specifies of biological tissue simultaneously with high resolution. In addition, ICG is needed to be injected into tail vein for fluorescence imaging. For integrated PA/US imaging. For integrated PA/US, the architectural morphology and vasculature of the rectum wall were visualized without the usage of any contrast agent. Compared with integrated OCT/NIR fluorescence imaging, the imaging speed is lower and match medium is necessary, whic are the main limitations for clinical translation. In summary, endoscopic multimodality imaging has shown to have strong potential to provide a new insight into the pathology of GI disease, which considerably reduce the cost and time of imaging compared to one of these imaging technologies alone.

\section{Acknowledgments}

This work was supported by grants from the National Institutes of Health (R01HL-125084, R01HL127271, R01EY-026091, R01EY-021529, and P41EB-015890) and the Air Force Office of Scientific Research (FA9550-17-1-0193). Dr. Chen has a financial interest in OCT Medical Imaging, Inc., which, however, did not support this work. 


\section{Funding}

National Institutes of Health (R01HL-125084, R01HL-127271, R01EY-026091, R01EY-021529, and P41EB-015890); Air Force Office of Scientific Research (FA9550-17-1-0193).

\section{References}

1. McGuire, S., World Cancer Report 2014. Geneva, Switzerland: World Health Organization, International Agency for Research on Cancer, WHO Press, 2015. Adv Nutr, 2016. 7(2): p. 418-9.

2. Kronborg, O., et al., Randomised study of screening for colorectal cancer with faecal-occult-blood test. Lancet, 1996. 348(9040): p. 1467-71.

3. Gostout, C.J. and S.L. Jacques, Infrared video imaging of subsurface vessels: a feasibility study for the endoscopic management of gastrointestinal bleeding. Gastrointest Endosc, 1995. 41(3): p. 218-24.

4. Harewood, G.C., Assessment of clinical impact of endoscopic ultrasound on rectal cancer. Am J Gastroenterol, 2004. 99(4): p. 623-7.

5. Iseki, K., et al., Effectiveness of the near-infrared electronic endoscope for diagnosis of the depth of involvement of gastric cancers. Gastrointest Endosc, 2000. 52(6): p. 755-62.

6. Yuan, S., et al., Co-registered optical coherence tomography and fluorescence molecular imaging for simultaneous morphological and molecular imaging. Phys Med Biol, 2010. 55(1): p. 191-206.

7. Li, Y., et al., High-speed intravascular spectroscopic photoacoustic imaging at 1000 A-lines per second with a 0.9-mm diameter catheter. Journal of Biomedical Optics, 2015. 20(6).

8. $\quad \mathrm{Li}, \mathrm{Y}$. , et al., Fully integrated optical coherence tomography, ultrasound, and indocyanine greenbased fluorescence tri-modality system for intravascular imaging. Biomedical Optics Express, 2017. 8(2): p. 1036-1044.

9. Qu, Y.Q., et al., Miniature probe for mapping mechanical properties of vascular lesions using acoustic radiation force optical coherence elastography. Scientific Reports, 2017. 7.

10. Mascagni, D., et al., Endoluminal ultrasound for early detection of local recurrence of rectal cancer. Br J Surg, 1989. 76(11): p. 1176-80.

11. Wang, X.D., et al., Noninvasive laser-induced photoacoustic tomography for structural and functional in vivo imaging of the brain. Nature Biotechnology, 2003. 21(7): p. 803-806.

12. Yao, J., K.I. Maslov, and L.V. Wang, In vivo photoacoustic tomography of total blood flow and potential imaging of cancer angiogenesis and hypermetabolism. Technol Cancer Res Treat, 2012. 11(4): p. 301-7.

13. Standish, B.A., et al., In vivo endoscopic multi-beam optical coherence tomography. Phys Med Biol, 2010. 55(3): p. 615-22.

14. Tsai, T.H., et al., Endoscopic optical coherence angiography enables 3-dimensional visualization of subsurface microvasculature. Gastroenterology, 2014. 147(6): p. 1219-21.

15. Yang, V.X., et al., Endoscopic Doppler optical coherence tomography in the human GI tract: initial experience. Gastrointest Endosc, 2005. 61(7): p. 879-90.

16. Chen, Z., et al., Optical Doppler tomographic imaging of fluid flow velocity in highly scattering media. Opt Lett, 1997. 22(1): p. 64-6.

17. Lee, A.M.D., et al., In vivo lung microvasculature visualized in three dimensions using fiber-optic color Doppler optical coherence tomography. Journal of Biomedical Optics, 2013. 18(5).

18. Alander, J.T., et al., A review of indocyanine green fluorescent imaging in surgery. Int J Biomed Imaging, 2012. 2012: p. 940585. 
19. Kim, S.Y. and S.J. Myung, Optical molecular imaging for diagnosing intestinal diseases. Clin Endosc, 2013. 46(6): p. 620-6.

20. Kimura, T., et al., Infrared fluorescence endoscopy for the diagnosis of superficial gastric tumors. Gastrointest Endosc, 2007. 66(1): p. 37-43. 